\title{
ERRATA CORRIGE
}

\section{Weak Pareto Optimality of Multiobjective Problems in a Locally Convex Linear Topological Space}

\author{
M. MINAMI \\ Communicated by P. Varaiya
}

\begin{abstract}
Several corrections to Ref. 1 are pointed out.
\end{abstract}
Key Words. Multiobjective programs, weak Pareto optimality, cones of directions, polar cone, convexity, subdifferential.

With reference to the paper published in Ref. 1, the following corrections should be made.

(a) The assumptions int $\Omega_{i} \neq \varnothing$ and int $\Lambda=\varnothing$ of Problem 2.1 (page 470) should be omitted.

(b) All convex cones $K_{i}$ in Lemma 2.1 (page 472) and Theorem 2.1 (page 473) should be assumed to be nonempty. ${ }^{2}$ 475):

(c) The following assumption should be added to Problem 3.1 (page

$$
f_{i}\left(x_{0}\right)>\inf _{x \in X} f_{i}(x), \quad \text { for each } i=1,2, \ldots, m,
$$

and TC $\left(x_{0}, \Lambda\right)$ is a nonempty subset in $X$.

(d) The sentence in lines 15-20 of page 476, starting with "and that, for $i$ such that" and ending with " $K_{n+i}^{\circ}=X^{*}$ " should be omitted.

(e) The expression $\min _{x \in X} f_{i}(x)$ on line 12 of page 476 , line 13 of page 482 , and line 1 from the bottom of page 483 should be replaced by the expression $\inf _{x \in X} f_{i}(x)$.

\footnotetext{
${ }^{1}$ Associate Professor, Department of Management, Faculty of Economics, Yamaguchi University, Yosida, Yamaguchi, Japan.

${ }^{2}$ The author is indebted to Dr. Y. Nagahisa for pointing this out.
} 


\section{References}

1. Minami, M., Weak Pareto Optimality of Multiobjective Problems in a Locally Convex Linear Topological Space, Journal of Optimization Theory and Applications, Vol. 34, No. 4, 1981. 